\title{
ESTIMATION OF PHOSPHORUS DISTRIBUTION RATIO AT THE END OF BLOWING IN BOF
}

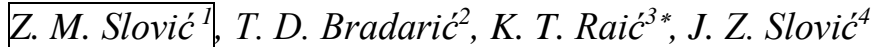 \\ ${ }^{1}$ Key to Metals Ltd., Belgrade, Serbia \\ ${ }^{2}$ KOTEH Engineering Ltd., Smederevo, Serbia \\ ${ }^{3}$ University of Belgrade, Faculty of Technology and Metallurgy, Serbia \\ ${ }^{4}$ Holycode, Belgrade, Serbia
}

Received 14.05.2021

Accepted 11.12.2021

\begin{abstract}
In integrated steel plants, the removal of phosphorous normally takes place during the primary basic oxygen furnace (BOF) steelmaking process. Phosphorous is usually introduced to the integrated steelmaking process through blast furnace additions, such as iron ore, coke, sinter, and fluxes. Among the others parameters such as optimizing the charging system, oxygen supply system, oxygen lance parameters of the converter, the flux quality in combination with temperature process control can improve the BOF efficiency of Dephosphorization. Phosphorus partition ratio $\left(\mathrm{L}_{\mathrm{P}}\right)$ is usually used to evaluate the thermodynamic efficiency of the dephosphorization of slags with different compositions in steelmaking processes. However, this parameter is only useful in equilibrium conditions, and it is not accurate when used to evaluate slag efficiency in industrial processes. Because of this, the aim of this work was to study the phosphorus partition ratio estimated from the experimental results in real plant conditions of two different BOF steel plants and compare them with well-known published models. In the present study, data from two steel plants (further Plant A and Plant B) were evaluated applying Healy's, Suito and Inoui's, Zhang's as well as Assis's equations. The calculated values were compared against measured values.
\end{abstract}

Keywords: BOF; phosphorus distribution ratio; dephosphorization; activity coefficient of $\mathrm{P}_{2} \mathrm{O}_{5}$. 


\section{Introduction}

A long time ago, phosphorus was recognized as a deleterious trace element in steel, which must be strictly controlled. Reducing phosphorus content in the steel is one of the critical factors to enhance the steel properties. In recent times, lowering phosphorus content has become a critical requirement for steels used in thin sheets for deep drawn applications, automobile exteriors, pipelines for transporting natural gas and petroleum products, or heavy plates for shipbuilding.

Phosphorous is primarily introduced to the integrated steelmaking process through blast furnace additions, such as iron ore, coke, sinter, and fluxes [1-3]. Therefore, raw material selection is crucial in maintaining the desired phosphorous level in the final product [4].

\section{Effect of phosphorus on the properties of steels}

Steels containing phosphorous can have good and bad properties. Phosphorous has a significant influence on the solid solution strengthening of the ferrite. The addition of a modest quantity of phosphorous can increase the yield and ultimate strength of the mild steel. It also increases the deep drawability and hardness of steel. Referable to the above advantages, such steels are used for cold forming operations. Phosphorous in steel can also improve corrosion resistance and metal cutting characteristics [5]. Table 1 summarizes the impact of phosphorous on the various steel properties.

Table 1. Effects of phosphorus on properties of steels [6].

\begin{tabular}{lll}
\hline & Property & Effect of phosphorus \\
\hline 1 & Strength & Strong positive (strengthens ferrite) \\
2 & Bake hardenability & Positive \\
3 & Ductility & Strong negative \\
4 & Galvannealing & Can improve resistance to powdering \\
5 & Phosphatability & Positive \\
6 & Enameling & \\
& a. Fish scaling & Negative \\
& b. Pickling & Positive \\
7 & Weldability & Not harmful for $[\% \mathrm{P}]<0.1$ \\
8 & Core loss in motor lamination & Strong negative \\
9 & Fracture toughness & Strong negative \\
\hline
\end{tabular}

The most common form widely used as a measure of slag's ability to remove phosphorus from the melt is phosphorus partition ratio between slag and metal, mathematically expressed [4], applying Equation (1):

$$
L_{P}=\frac{\left(\% P_{2} O_{5}\right)}{[\% P]}
$$

In the available literature, many correlations have been developed to predict the phosphorus partition ratio as a function of metal and slag composition as well as temperature. However, there are still disagreements between the laboratory and calculated data on the equilibrium phosphorus partition ratio [6].

Although the quantification of phosphorus equilibrium is extremely important, many steelmaking furnaces do not operate at equilibrium, usually due to liquid slag 
formation, kinetics and time constraints. Thus, it is important to know how close to equilibrium steelmaking furnaces operate to suggest optimal slag compositions to promote dephosphorization [6].

A number of studies in the past have been carried out to develop predictive models for the equilibrium partition ratio of phosphorus (Lp) as a function of slag chemistry and temperature [4,7-18]. Such models use empirical regression equations of the measured equilibrium data to estimate the Lp. Recently Drain et al. carried out a critical assessment of more than 90 different phosphorus partition models with the industrial converter data and proposed a new relationship that can be applied for a wide range of slag constituents [19]. Several phosphorus distribution ratio models are illustrated in Table 2. Healy [13] proposed an empirical correlation for phosphorus distribution as a function of slag composition and temperature. This correlation was suggested for $\mathrm{CaO}-\mathrm{SiO}_{2}-\mathrm{Fe}_{\mathrm{t}} \mathrm{O}$ type of slag with high content of $\mathrm{CaO}$ and $\mathrm{Fe}_{\mathrm{t}} \mathrm{O}$. Turkdogan [10] critically assessed the slag-metal equilibrium for simple and complex slags based on the previous laboratory scale experiments and derived a new partition ratio for phosphorus. In the 1980s, Suitoet al. [8] derived a correlation for phosphorus distribution in $\mathrm{MgO}$-saturated slags of $\mathrm{CaO}-\mathrm{FetO}-\mathrm{SiO}_{2}-\mathrm{P}_{2} \mathrm{O}_{5}-\mathrm{MnO}$ system. The correlation is based on the ionic concept developed by Healy. Additionally, Ide and Fruehan [12] varied the $\mathrm{MgO}$ concentration in their experiments and proposed a revised version of Suito's correlation for equilibrium phosphorus distribution [4].

It is well known that, the calculation of theoretical phosphorus distribution ratios Lp is difficult due to the fact that dephosphorization reaction in the BOF at EOB is under non-equilibrium condition. According to results published in the earlier mentioned studies [4, 7-13, 20-24], the empirical formulas for calculating Lp are summarized in Table 2 .

In this work, some models from Table 2 and 3 have been used to determine the phosphorus partition coefficient and activity coefficient of $\mathrm{P}_{2} \mathrm{O}_{5}\left(\log \mathrm{YP}_{2} \mathrm{O}_{5}\right)[25,26]$, based on classic thermodynamic approach.

Table 2. Various formulas to describe the phosphorus partition ratio.

\begin{tabular}{|c|c|c|}
\hline $\begin{array}{l}\text { Lp } \\
\text { models }\end{array}$ & Formulas of the Lp models & Ref. \\
\hline Healy & $\log (\% \mathrm{P}) /[\% \mathrm{P}]=22350 / \mathrm{T}+0.08(\% \mathrm{CaO})+2.5 \log (\% \mathrm{Fet})-16 \pm 0.4$ & {$[1,13]$} \\
\hline $\begin{array}{l}\text { Suito- } \\
\text { Inoue }\end{array}$ & $\begin{array}{l}\log (\% \mathrm{P}) /[\% \mathrm{P}]=0.0720\left[(\% \mathrm{CaO})+0.022(\% \mathrm{MgO})+0.043\left(\% \mathrm{P}_{2} \mathrm{O}_{5}\right)+\right. \\
0.014(\% \mathrm{MnO})+2.5 * \log (\% \mathrm{Fet})+11570 / \mathrm{T}-10.520\end{array}$ & {$[1,20]$} \\
\hline Zhang & $\begin{array}{l}\log (\% \mathrm{P}) /[\% \mathrm{P}]=11000 / \mathrm{T}+(1 / \mathrm{T}) *(162 * \% \mathrm{CaO}+127.5 * \% \mathrm{MgO}+28.5 \\
* \% \mathrm{MnO})+ \\
2.5 * \log (\% \mathrm{FeO})-0.000628 * \mathrm{SiO}_{2}-10.76\end{array}$ & [21] \\
\hline Assis & $\begin{array}{l}\log (\% \mathrm{P}) /[\% \mathrm{P}]=0.073[(\% \mathrm{CaO})+0.148(\% \mathrm{MgO})+0.8(\% \mathrm{P} 2 \mathrm{O} 5) \\
\left.0.113\left(\% \mathrm{SiO}_{2}\right)\right]+2.5 \log \left(\mathrm{Fe}_{\mathrm{T}}\right)+11570 / \mathrm{T}-10.403\end{array}$ & - $[22]$ \\
\hline
\end{tabular}

Formulas for calculation of activity coefficient of $\mathrm{P}_{2} \mathrm{O}_{5}\left(\log \mathrm{Y}_{\mathrm{P} 2 \mathrm{O} 5}\right)$ used in this work are shown in Table3. 
Table 3. Equations for prediction of activity coefficient of $\mathrm{P}_{2} \mathrm{O}_{5}\left(\log Y_{P 2 O 5}\right)$.

\begin{tabular}{lll}
\hline & Formulas & Ref. \\
\hline Mori & $9.4-38.09 * \Lambda$ & {$[24]$} \\
Turkdogan & $-34850 / \mathrm{T}-3.85-0.058(\% \mathrm{CaO})$ for $\mathrm{P}_{2} \mathrm{O}_{5}<10 \mathrm{wt} . \%$ & {$[10,23]$} \\
\hline
\end{tabular}

\section{Experimental procedure}

The present investigations were conducted at two different steelmaking plants using blast furnace-basic oxygen steelmaking (BF-BOF) to produce liquid steel. External Dephosphorization through hot metal pre-treatment was not used. The target for the production of low phosphorus steel was achieved by single-stage blowing of hot metal in the BOF. The production was tested with a 100t Top-Blowing BOF of 25 heats(Steel-Plant A) [25] and 180t Combined blowing BOF of 14 heats (Steel-Plant B) [26] in the further marked as Plant A and Plant B. The composition and temperature of steel and slag are shown in Tables 4 and 5.

Table 4. Chemical composition of analyzed steel in Plant A and Plant B.

\begin{tabular}{|c|c|c|c|c|c|c|c|c|c|c|}
\hline \multirow[b]{2}{*}{ Heat } & \multicolumn{4}{|c|}{ Plant A } & \multirow[b]{2}{*}{$\boldsymbol{\eta}_{\boldsymbol{P}} \mathrm{T},\left[{ }^{\circ} \mathrm{C}\right]$} & \multirow[b]{2}{*}{$\mathrm{P}_{\mathrm{HM}}$} & \multirow[b]{2}{*}{$\mathrm{C}$} & \multicolumn{2}{|l|}{ Plant B } & \multirow[b]{2}{*}{$\boldsymbol{\eta}_{\boldsymbol{P}} \mathrm{T},\left[{ }^{\circ} \mathrm{C}\right]$} \\
\hline & $\mathrm{P}_{\mathrm{HM}}$ & $\mathrm{C}$ & $\mathrm{Mn}$ & $\mathrm{P}$ & & & & $\mathrm{Mn}$ & $\mathrm{P}$ & \\
\hline 1 & 0.07 & 0.031 & 0.100 & 0.012 & 831680 & 0.114 & 0.0182 & 0.0760 & 0.0065 & 941710 \\
\hline 2 & 0.07 & 0.032 & 0.050 & 0.007 & 901666 & 0.104 & 0.0248 & 0.1110 & 0.0076 & 931704 \\
\hline 3 & 0.05 & 0.040 & 0.080 & 0.006 & 881653 & 0.089 & 0.0195 & 0.1210 & 0.0089 & 901722 \\
\hline 4 & 0.05 & 0.029 & 0.085 & 0.006 & 881648 & 0.098 & 0.0208 & 0.1640 & 0.0109 & 891707 \\
\hline 5 & 0.07 & 0.041 & 0.091 & 0.006 & 911662 & 0.094 & 0.0203 & 0.0760 & 0.0060 & 941696 \\
\hline 6 & 0.07 & 0.082 & 0.090 & 0.005 & 931657 & 0.090 & 0.0247 & 0.1360 & 0.0095 & 891695 \\
\hline 7 & 0.06 & 0.048 & 0.070 & 0.007 & 881644 & 0.095 & 0.0331 & 0.1850 & 0.0112 & 881720 \\
\hline 8 & 0.06 & 0.030 & 0.110 & 0.011 & 821656 & 0.098 & 0.0270 & 0.1140 & 0.0078 & 921720 \\
\hline 9 & 0.06 & 0.026 & 0.133 & 0.011 & 821664 & 0.106 & 0.0500 & 0.1670 & 0.0104 & 901718 \\
\hline 10 & 0.06 & 0.023 & 0.121 & 0.011 & $82 \quad 1680$ & 0.099 & 0.0264 & 0.1190 & 0.0104 & $89 \quad 1713$ \\
\hline 11 & 0.06 & 0.032 & 0.103 & 0.009 & 851658 & 0.098 & 0.0179 & 0.1050 & 0.0089 & 911696 \\
\hline 12 & 0.06 & 0.028 & 0.110 & 0.013 & 781666 & 0.089 & 0.0391 & 0.1180 & 0.0083 & 911681 \\
\hline 13 & 0.06 & 0.021 & 0.070 & 0.010 & 831670 & 0.094 & 0.0267 & 0.1440 & 0.0197 & 791688 \\
\hline 14 & 0.07 & 0.039 & 0.122 & 0.009 & 871659 & 0.106 & 0.0377 & 0.2070 & 0.0201 & 811716 \\
\hline 15 & 0.07 & 0.043 & 0.092 & 0.008 & 891658 & 0.096 & 0.0244 & 0.1540 & 0.0253 & 741719 \\
\hline 16 & 0.07 & 0.035 & 0.090 & 0.010 & 861677 & 0.099 & 0.0505 & 0.2260 & 0.0265 & 731715 \\
\hline 17 & 0.06 & 0.030 & 0.105 & 0.011 & 821658 & & & & & \\
\hline 18 & 0.07 & 0.018 & 0.120 & 0.016 & 771660 & & & & & \\
\hline 19 & 0.07 & 0.020 & 0.290 & 0.024 & 661647 & & & & & \\
\hline 20 & 0.07 & 0.028 & 0.132 & 0.020 & 711659 & & & & & \\
\hline 21 & 0.07 & 0.031 & 0.089 & 0.006 & 911649 & & & & & \\
\hline 22 & 0.07 & 0.034 & 0.077 & 0.007 & 901656 & & & & & \\
\hline 23 & 0.06 & 0.043 & 0.078 & 0.007 & 881657 & & & & & \\
\hline 24 & 0.05 & 0.028 & 0.120 & 0.018 & 641678 & & & & & \\
\hline 25 & 0.06 & 0.029 & 0.099 & 0.008 & $87 \quad 1641$ & & & & & \\
\hline
\end{tabular}


Table 5. Slag chemical composition of analyzed heats, wt.\%.

\begin{tabular}{|c|c|c|c|c|c|c|c|c|c|c|c|c|}
\hline \multirow[b]{2}{*}{ Heat } & \multicolumn{5}{|c|}{ Plant A } & \multicolumn{7}{|c|}{ Plant B } \\
\hline & $\mathrm{CaO}$ & $\mathrm{SiO}_{2}$ & Fe tot. & $\mathrm{MnO}$ & $\mathrm{MgO}$ & $\mathrm{P}_{2} \mathrm{O}_{5}$ & $\mathrm{CaO}$ & $\mathrm{SiO}_{2}$ & Fe tot. & $\mathrm{MnO}$ & $\mathrm{MgO}$ & $\mathrm{P}_{2} \mathrm{O}_{5}$ \\
\hline 1 & 49.34 & 13.91 & 26.21 & 5.02 & 4.08 & 1.21 & 46.24 & 7.02 & 26.0 & 2.10 & 1.47 & 1.68 \\
\hline 2 & 36.40 & 9.59 & 30.68 & 4.81 & 3.98 & 0.71 & 47.93 & 10.12 & 22.9 & 2.93 & 1.33 & 1.59 \\
\hline 3 & 42.79 & 12.39 & 20.40 & 5.07 & 3.73 & 0.87 & 43.07 & 10.47 & 25.5 & 3.40 & 2.20 & 1.65 \\
\hline 4 & 45.83 & 12.34 & 19.66 & 4.58 & 5.24 & 0.80 & 51.18 & 11.35 & 18.7 & 3.40 & 1.65 & 1.79 \\
\hline 5 & 51.91 & 15.28 & 14.10 & 4.48 & 4.87 & 0.98 & 37.85 & 6.12 & 34.7 & 2.88 & 2.69 & 1.21 \\
\hline 6 & 49.52 & 15.29 & 15.02 & 4.54 & 4.15 & 0.88 & 48.12 & 11.12 & 20.5 & 3.61 & 3.32 & 1.67 \\
\hline 7 & 47.13 & 12.90 & 19.93 & 4.21 & 3.62 & 0.94 & 50.29 & 10.82 & 19.6 & 3.96 & 1.85 & 1.66 \\
\hline 8 & 40.62 & 20.87 & 15.48 & 4.75 & 4.43 & 0.69 & 50.13 & 9.06 & 21.5 & 2.93 & 3.36 & 1.07 \\
\hline 9 & 45.58 & 19.30 & 13.04 & 5.76 & 3.05 & 0.92 & 61.67 & 14.76 & 10.8 & 2.18 & 1.44 & 1.14 \\
\hline 10 & 50.92 & 16.29 & 12.40 & 5.44 & 2.92 & 0.93 & 45.87 & 10.55 & 24.2 & 3.27 & 1.45 & 2.01 \\
\hline 11 & 49.06 & 14.33 & 15.39 & 5.04 & 3.21 & 0.93 & 40.96 & 8.34 & 29.1 & 3.40 & 1.66 & 2.02 \\
\hline 12 & 46.59 & 14.76 & 14.34 & 5.18 & 3.55 & 0.90 & 55.27 & 6.78 & 22.8 & 2.54 & 1.55 & 1.42 \\
\hline 13 & 49.08 & 17.43 & 13.16 & 5.70 & 3.22 & 0.84 & 54.67 & 6.54 & 21.2 & 2.45 & 1.51 & 1.56 \\
\hline 14 & 48.85 & 15.31 & 13.10 & 5.15 & 3.49 & 0.99 & 56.06 & 13.58 & 13.9 & 3.06 & 1.70 & 2.31 \\
\hline 15 & 50.91 & 14.38 & 15.47 & 5.01 & 2.69 & 0.95 & 49.59 & 12.69 & 19.4 & 3.60 & 1.25 & 2.73 \\
\hline 16 & 48.78 & 12.34 & 19.37 & 4.96 & 2.81 & 0.92 & 56.49 & 14.55 & 13.7 & 2.90 & 1.58 & 2.34 \\
\hline 17 & 50.34 & 14.87 & 12.47 & 5.22 & 3.40 & 1.03 & & & & & & \\
\hline 18 & 50.08 & 15.26 & 11.61 & 5.41 & 2.71 & 1.11 & & & & & & \\
\hline 19 & 51.67 & 15.28 & 11.42 & 5.31 & 2.74 & 1.09 & & & & & & \\
\hline 20 & 52.52 & 15.74 & 11.64 & 5.51 & 2.78 & 1.12 & & & & & & \\
\hline 21 & 46.38 & 11.53 & 19.04 & 5.08 & 3.42 & 1.07 & & & & & & \\
\hline 22 & 44.35 & 10.22 & 19.82 & 5.53 & 3.9 & 0.31 & & & & & & \\
\hline 23 & 40.74 & 8.41 & 24.21 & 4.98 & 3.54 & 0.86 & & & & & & \\
\hline 24 & 46.27 & 14.71 & 14.85 & 6.29 & 3.7 & 1.3 & & & & & & \\
\hline 25 & 47.33 & 13.9 & 15.46 & 5.3 & 4.53 & 1.09 & & & & & & \\
\hline
\end{tabular}

\section{Results and discussion}

The removal of phosphorus from the furnace charge is a well understood process. It can be described by the DePhos coefficient $\mathrm{L}_{\mathrm{P}}=\left(\% \mathrm{P}_{2} \mathrm{O}_{5}\right) /[\% \mathrm{P}]$, which represents the distribution between slag $-\left(\% \mathrm{P}_{2} \mathrm{O}_{5}\right)$, and metal $-[\% \mathrm{P}]$. It is well known that the $\mathrm{L}_{\mathrm{P}}$ depends on many process parameters such as temperature of the melt, slag ferrous content $\left(\% \mathrm{Fe}_{\text {tot. }}\right)$, slag $\mathrm{V}$-ratio $(\% \mathrm{CaO}) /\left(\% \mathrm{SiO}_{2}\right),(\% \mathrm{MgO})$, refractory wear, campaign life, top or combined blowing BOF process, flux quality, slag volume, etc.

The obtained results of our study are listed in Tables 6 and 7. 
Table 6. The log Lpmeasure vs. log Lp calculated applying various models.

\begin{tabular}{|c|c|c|c|c|c|c|c|c|c|c|}
\hline Heat & Lp m. & Healy & $\begin{array}{l}\text { Plant } \\
\text { A } \\
\text { Zhang }\end{array}$ & $\begin{array}{l}\text { Suito- } \\
\text { Inoue }\end{array}$ & Assis & Lp m & Healy & $\begin{array}{l}\text { Zhan } \\
\text { g }\end{array}$ & $\begin{array}{l}\text { Suito- } \\
\text { Inoue }\end{array}$ & Assis \\
\hline 1 & 2.00 & 2.94 & 2.84 & 2.68 & 2.90 & 2.41 & 2.51 & 2.22 & 2.17 & 2.52 \\
\hline 2 & 2.01 & 2.16 & 2.00 & 1.85 & 2.10 & 2.32 & 2.54 & 2.25 & 2.02 & 2.54 \\
\hline 3 & 2.16 & 2.30 & 2.14 & 1.88 & 2.20 & 2.27 & 2.16 & 1.95 & 1.93 & 2.26 \\
\hline 4 & 2.12 & 2.53 & 2.47 & 2.18 & 2.40 & 2.22 & 2.56 & 2.31 & 1.94 & 2.57 \\
\hline 5 & 2.21 & 2.58 & 2.52 & 2.23 & 2.47 & 2.30 & 2.23 & 2.00 & 1.93 & 2.24 \\
\hline 6 & 2.25 & 2.48 & 2.37 & 2.03 & 2.37 & 2.24 & 2.49 & 2.33 & 1.91 & 2.49 \\
\hline 7 & 2.13 & 2.68 & 2.50 & 2.24 & 2.52 & 2.17 & 2.47 & 2.25 & 1.86 & 2.51 \\
\hline 8 & 1.80 & 1.81 & 1.68 & 1.48 & 1.79 & 2.14 & 2.56 & 2.42 & 1.90 & 2.57 \\
\hline 9 & 1.92 & 1.97 & 1.79 & 1.63 & 1.93 & 2.04 & 2.74 & 2.48 & 1.53 & 2.70 \\
\hline 10 & 1.93 & 2.25 & 2.09 & 1.87 & 2.19 & 2.29 & 2.38 & 2.11 & 1.99 & 2.45 \\
\hline 11 & 2.01 & 2.47 & 2.30 & 2.06 & 2.35 & 2.36 & 3.09 & 2.81 & 2.17 & 2.97 \\
\hline 12 & 1.84 & 2.15 & 2.00 & 1.71 & 2.07 & 2.06 & 2.58 & 2.34 & 1.79 & 2.63 \\
\hline 13 & 1.92 & 2.23 & 2.08 & 1.83 & 2.16 & 2.03 & 2.41 & 2.14 & 1.92 & 2.52 \\
\hline 14 & 2.04 & 2.27 & 2.12 & 1.80 & 2.17 & 1.95 & 2.60 & 2.35 & 1.77 & 2.66 \\
\hline 15 & 2.07 & 2.62 & 2.42 & 2.19 & 2.48 & & & & & \\
\hline 16 & 1.96 & 2.58 & 2.40 & 2.20 & 2.49 & & & & & \\
\hline 17 & 1.97 & 2.34 & 2.19 & 1.89 & 2.22 & & & & & \\
\hline 18 & 1.84 & 2.23 & 2.04 & 1.82 & 2.12 & & & & & \\
\hline 19 & 1.66 & 2.42 & 2.22 & 1.96 & 2.26 & & & & & \\
\hline 20 & 1.75 & 2.43 & 2.26 & 2.00 & 2.31 & & & & & \\
\hline 21 & 2.25 & 2.54 & 2.37 & 2.16 & 2.40 & & & & & \\
\hline 22 & 1.65 & 2.38 & 2.24 & 1.85 & 2.22 & & & & & \\
\hline 23 & 2.09 & 2.30 & 2.12 & 1.83 & 2.18 & & & & & \\
\hline 24 & 1.86 & 2.09 & 1.97 & 1.82 & 2.07 & & & & & \\
\hline 25 & 2.13 & 2.44 & 2.34 & 2.04 & 2.30 & & & & & \\
\hline
\end{tabular}


Table 7. Calculated values of the activity coefficient of $\mathrm{P}_{2} \mathrm{O}_{5}\left(\log Y_{P 2 O 5}\right)$, (see Table 3, and reference [25])

\begin{tabular}{lllll}
\hline & Plant A & & Plant B & \\
\hline Heat & $\begin{array}{l}\text { Turkdogan, for } \\
\% \mathrm{P}_{2} \mathrm{O}_{5}<10\end{array}$ & Mori & $\begin{array}{l}\text { Turkdogan, for } \\
\%_{2} \mathrm{O}_{5}<10\end{array}$ & Mori \\
\hline 1 & 24.56 & 19.30 & 24.11 & 18.84 \\
2 & 23.93 & 18.00 & 24.26 & 18.86 \\
3 & 24.43 & 18.60 & 23.82 & 18.36 \\
4 & 24.65 & 19.30 & 24.42 & 19.53 \\
5 & 24.87 & 19.70 & 23.74 & 17.85 \\
6 & 24.78 & 19.10 & 24.35 & 19.75 \\
7 & 24.76 & 19.30 & 24.25 & 20.63 \\
8 & 24.27 & 17.60 & 24.24 & 20.75 \\
9 & 24.49 & 18.30 & 24.93 & 18.44 \\
10 & 24.65 & 19.40 & 24.06 & 20.07 \\
11 & 24.74 & 19.40 & 24.79 & 19.96 \\
12 & 24.53 & 18.90 & 24.62 & 18.77 \\
13 & 24.63 & 18.80 & 24.22 & 19.87 \\
14 & 24.72 & 19.30 & 24.66 & \\
15 & 24.85 & 19.50 & & \\
16 & 24.55 & 19.40 & & \\
17 & 24.82 & 19.50 & & \\
18 & 24.78 & 19.40 & & \\
19 & 25.00 & 19.70 & & \\
20 & 24.93 & 19.80 & & \\
21 & 24.67 & 19.40 & & \\
22 & 24.49 & 19.10 & & \\
24 & 24.27 & 18.90 & & \\
\hline
\end{tabular}

Figures 1 and 2 show the comparison between measured and calculated phosphorus partition ratio (Lp) for Plant A and Plant B, applying Healy's, Suito, and Inoui's, Zhang's as well as Assis's equations. For Plant A, the predicted Lp values using Healy's equation are very close to the measured values. However, for Plant B, Healy's equation over predicts the Lp values by a factor of 2 to 3 . The over prediction by Healy's equation is a common observation and has been reported by others. The fact that Healy's equation predicts the phosphorus partition in Plant A very close to actual measured values can be attributed to the fact that Healy's equation may have been developed for low phosphorus hot metal based on the ionic theory of slags. 


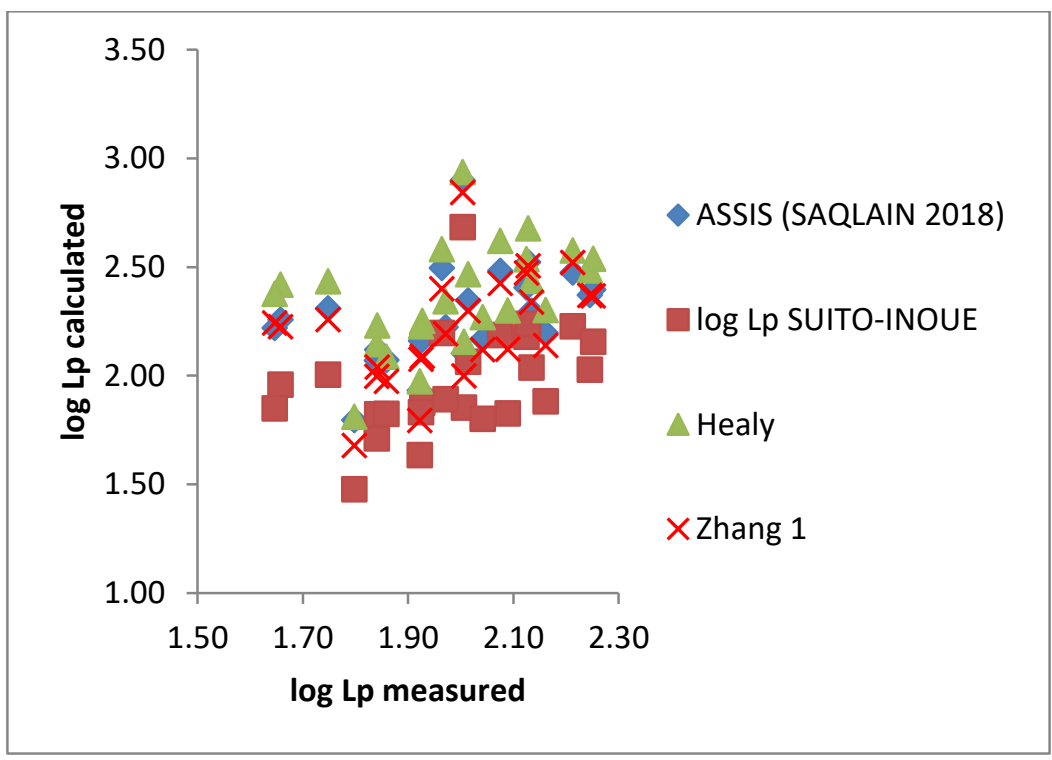

Fig. 1. Comparison of $\log L p$ values estimated using predicted log Lp equations with measured values at Plant $A$.

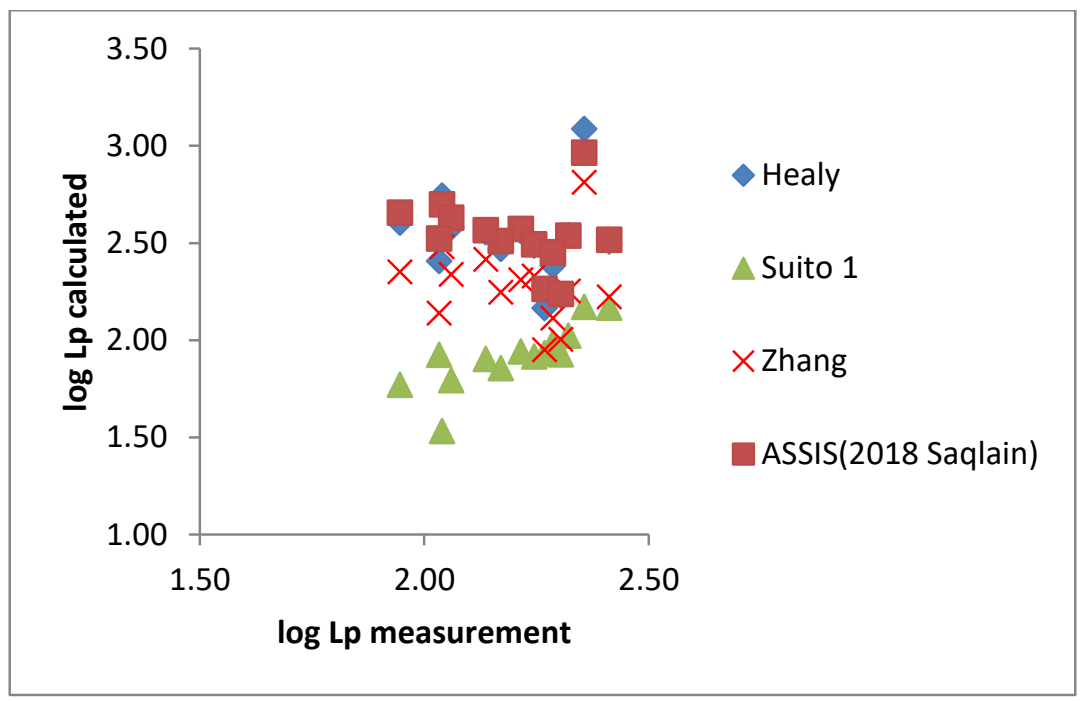

Fig. 2. Comparison of log Lp values estimated using predicted log Lp equations with measured values at Plant $B$. 
On the other hand, the dephosphorization rate (De-P rate) can be used as a key indicator, which can be calculated by Equation (2):

$$
\text { De }-P \text { rate }=100 \%\left(1-\frac{m_{1} P_{1}}{m_{2} P_{2}}\right)
$$

where,

De-Prate is the dephosphorization rate, $\% ; m_{1}$ and $m_{2}$ are the mass of steel before and after the treatment, respectively, $\mathrm{kg} ; P_{1}$ and $P_{2}$ are the phosphorus content before and after the treatment, respectively, mass\%.

Therefore, the correlations between calculated log Lp of literature models with DeP-rate, [\%] for Plant A and Plant B are given in Figures 3 and 4.

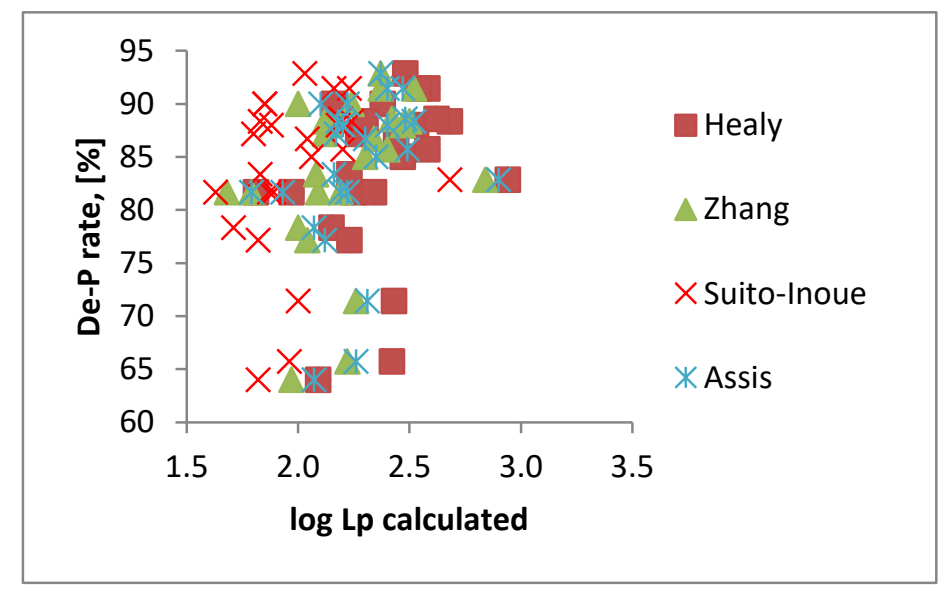

Fig. 3. Plant A: Correlation between calculated log Lp of literature models with DeP-rate, [\%].

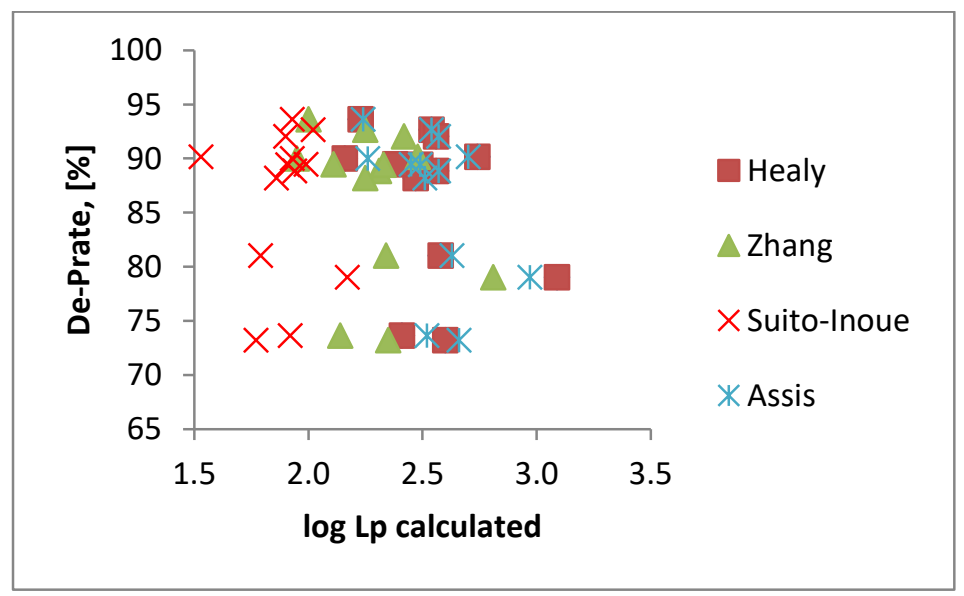

Fig. 4. Plant B: Correlation between calculated log Lp of literature models with DeP-rate, [\%]. 
Figures 5 and 6 have compared the activity coefficient of the $\mathrm{P}_{2} \mathrm{O}_{5}$ calculated using Turkdogan and Mori model for both Plants. It is evident that there is a stronger correlation in the activity coefficient of the $\mathrm{P}_{2} \mathrm{O}_{5}$ calculated from Plant $\mathrm{B} \log \mathrm{Y}_{\mathrm{P} 2 \mathrm{O}_{5}-}$ Turkdogan-log Y $\mathrm{P}_{205}$ Mori compared with correlation in Plant A.

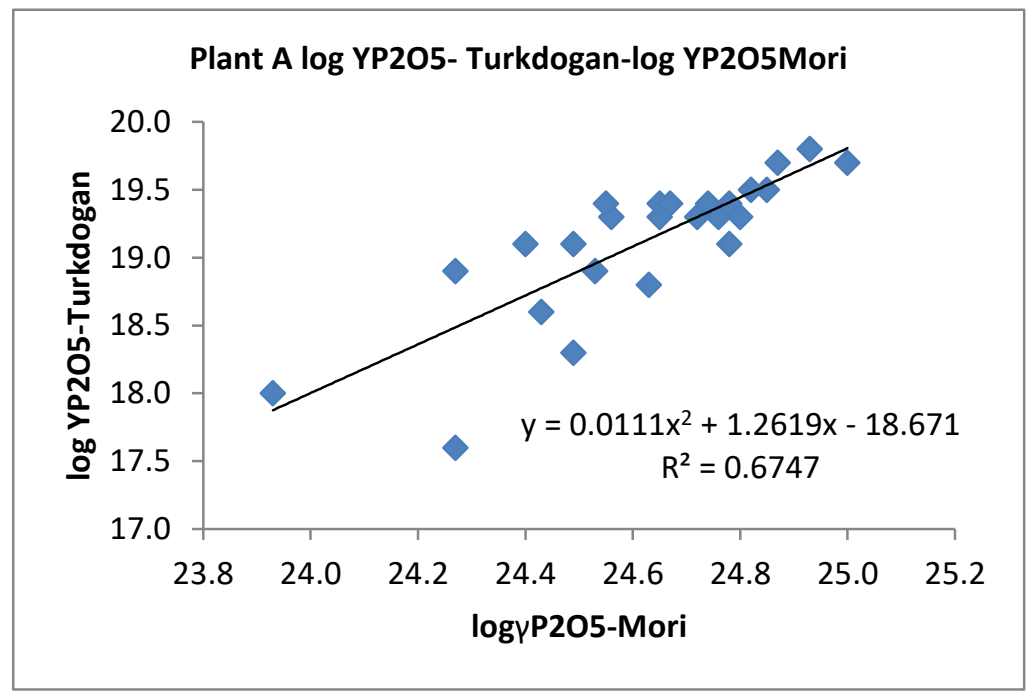

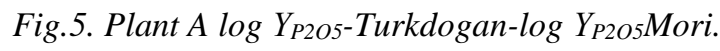

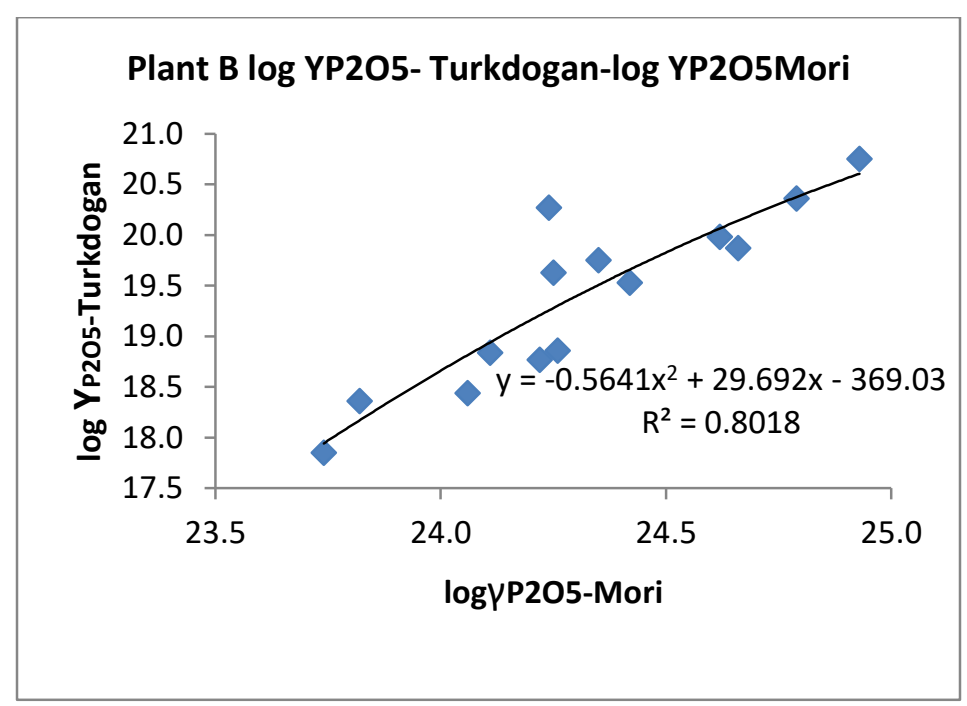

Fig. 6. Plant B log $Y_{P 2 O 5}$-Turkdogan-log $Y_{P 2 O 5}$ Mori. 


\section{Conclusions}

This study investigated the dephosphorization behavior of two different steel plants for a single slag practice by analyzing phosphorus distribution ratio LP and the activity coefficient of $\mathrm{P}_{2} \mathrm{O}_{5}$. According to the data of the two steel mills, one uses top blowing and the other uses top and bottom combined blowing. From the data in Table 4 and Table 5, there is little difference between the two. Consequently, these two types of blowing were taken to test the overall performance of the proposed procedure.

Based on industrial results, the conclusions are given as follows:

1. To achieve low phosphorus content in steel at the EOB in BOF, one needs low $\mathrm{P}$ input and high Lp is needed among the other parameters;

2. Analyzed models for calculation of Lp give a scattered value which confirms that the results are far from reaching equilibrium;

3. There is a stronger correlation in the activity coefficient of $\mathrm{P}_{2} \mathrm{O}_{5}\left(\log \mathrm{Y}_{\mathrm{P} 2 \mathrm{O}^{-}}\right.$ Turkdogan-log $\mathrm{Y}_{\mathrm{P} 205}$ Mori) calculated from Plant B compared with the correlation in Plant A.

4. There is a need to develop in further work and on much more analyzed heats, an equation that could be used as a predictive tool for estimating the phosphorus partition in steel plants.

\section{References}

[1] A. Adak, A. Das, S.Chatterjee, A. Senguttuvan, A. Mukherjee, Optimization of Dephosphorisation Practice in BOF Shops Using Advanced Analytics, Conference: AISTech, At: Pennsylvania, USA, 2018, https://www.researchgate.net/publication/327308125_Optimization_of_Dephosp horisation_Practice_in_BOF_Shops_Using_Advanced_Analytics/Accessed 26 April 2020/;Accessed 9. 2021.

[2] F. Costa Broseghini, H. Cristo Clem de Oliveria, S. GambarineSoares, F. FardinGrillo: REM, Int Eng J, 71 (2018) 217-223.

[3] K. Chattopadhyay, S. Kumar: Application of Thermodynamic Analysis for Developing Strategies to Improve BOF Steelmaking Process Capability, AISTech Conference Proceedings, Iron and Steel Technology AIST, Indiana Convention Center in Indianapolis, Ind., USA, 2014, PR-364-079 - 2013.

[4] B. Kumar Rout: Modelling of Dephosphorization in Oxygen Steelmaking, SWINBURNE UNIVERSITY OF TECHNOLOGY, Faculty of Science, Engineering and Technology Swinburne University of Technology, Melbourne, Australia March 2018, PhD Thesis, https://researchbank.swinburne.edu.au/items/28bcd64e-5f32-45c7-8befddf5063d95f5/1/; Accessed 9. 2021.

[5] A. Purohit: Dephosphorization of steel produced from sponge iron in the induction furnace, National Institute of Technology Rourkela-769008 May 2014, Roll No.-212MM2458, MSc Thesis, http://ethesis.nitrkl.ac.in/5856/1/212MM2458.pdf; Accessed 9. 2021.

[6] S. Kumar Sarna,Ispat Guru, 2016, Dephosphorization of steels, https://www.ispatguru.com/dephosphorization-of-steels/; Accessed 9. 2021.

[7] H. Suito, R. Inoue, M. Takada: Tetsu-to-Hagané 67 (16) (1981) 2645-2654.

[8] H. Suito, Hideaki, R. Inoue:Trans Iron Steel InstJpn, 24 (1) (1984) 47-53.

[9] H. Suito, R. Inoue: ISIJ International, 35(3) (1995) 258-265.

[10] E. T. Turkdogan: ISIJ International, 40(10) (2000) 964-970. 
[11] R. Inoue, H. Suito: ISIJ International, 46(2) (2006) 174-179.

[12] K. Ide, R.J. Fruehan: Iron \&steelmaker, 27(12) (2000) 65-70.

[13] G.W. Healy: JISI, 208 (1970) 664-668.

[14] W. Wu, Y.Qixing, G. A. O. Qi, J. Zeng:J Mater ResTechnol, 9 (2020) 27542761.

[15] F. He, Z.Lingying: J Process Control, 66 (2018) 51-58.

[16] B.K. Rout, G. Brooks, M.A.Rhamdhani, Z. Li, F.N.H.Schrama, J. Sun:Metall Mater Trans. B, 49 (2018) 537-557.

[17] K.X. Zhou, W.H.Lin, J.K.Sun, J.S.Zhang, D.Z. Zhang, X.M. Feng, Q.Liu:Journal of Iron and Steel Research International (2021) 1-10.

[18] S. Khadhraoui, H.J.Odenthal, S. Das, M.Schlautmann, K. Hack, B. Glaser, R. Woolf: La metallurgiaitaliana 11-12 (2018) 5-16.

[19] P.B. Drain, B.J. Monaghan, G. Zhang, R.J. Longbottom, M.W. Chapman, S.J. Chew: A review of phosphorus partition relations for use in basic oxygen steelmaking. Ironmaking \& Steelmaking, 44 (2017) 721-731.

[20] H. Suito, R. Inoue: ISIJ international, 35 (1995): 258-265.

[21] A.N. Assis, M.A. Tayeb, S. Seetharaman, R. Fruehan: Metall Mater Trans. B,46(2015)2255-2263.

[22] A.N. Assis: The Phosphorus Reaction in Oxygen Steelmaking: Thermodynamic Equilibrium and Metal Droplet Behavior, Carnegie Mellon University, Pittsburgh, Pennsylvania, USA, 2014, PhD Thesis, Paper 464./ https://pdfs.semanticscholar.org/9e7c/56424b1a9c8fa7078c62893ec7325cc9b572 .pdf?_ga=2.42388065.596048088.1592891855-2064382242.1542610121;

Accessed 9. 2021.

[23] M. Saqlain, M. Owais, M. Järvinen, V.V. Visuri, T. Fabritius, Desphosphorization in ironmaking and oxygen steelmaking, 2018; https://aaltodoc.aalto.fi/bitstream/handle/123456789/32161/J_saqlain_muhamm ad 2018.pdf?sequence\%5cu003d1\%5cu0026isAllowed\%5cu003dy; Accessed 9. 2021.

[24] T. Mori: Trans JpnInst Met, 25(11) (1984) 761-771.

[25] Z. Slović: Control of the process of removal of sulfur and phosphorus from metals in steel production, MSc thesis, TMF, University of Belgrade, Serbia, 2004, /in Serbian/;

[26] Z. Slović:Thermodynamic approach to desulfurization in out-of-furnace processing of oxygen-converter steel, $\mathrm{PhD}$ thesis, TMF, UniversityofBelgrade, Serbia, 2004, /inSerbian/; UDK: 669.01/.09 /18.12.2013.

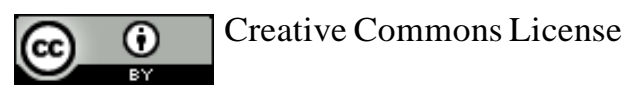

This work is licensed under a Creative Commons Attribution 4.0 International License. 